\title{
Achieving Treatment Free Prolonged Response in ITP patients Treated with Thrombopoietin Receptor Agonists (TPO-RAs)
}

\author{
Lunqing Zhang ${ }^{1}$, Mingjie Zhang ${ }^{1}$ and Gregory Cheng ${ }^{* 1,2,3}$ \\ ${ }^{1}$ Faculty of Health Science, University of Macau, Macau, China \\ ${ }^{2}$ Macau University of Science and Technology hospital, Macau, China \\ ${ }^{3}$ Health and Humanity Research Centre, Hongkong
}

${ }^{\star}$ Corresponding author: Gregory Cheng, Humanity and Health Clinical Trial Center, Humanity \& Health Medical Group, Hong Kong SAR, China; E-mail: gregory.cheng@hnhmgl.com

Received: May 10, 2020; Accepted: May 20, 2020; Published: May 25, 2020

\section{Mini Review}

Over the past ten years, Thrombopoietin Receptor Agonists (TPO-RAs) have become a popular treatment choice for Immune Thrombocytopenia (ITP) patients who have either failed or suffered from side effects of steroids therapy. Currently, three TPO-RAs have been licensed for the treatment of ITP, namely Romiplostim (Nplate, Amgen, Thousand Oaks, CA, USA), eltrombopag (Promacta, Novartis, Basel, Switzerland) and avatrombopag (Doptelet, Dova, Durham, NC, USA). Romiplostim is administered subcutaneously on a weekly schedule [1], while both eltrombopag and avatrombopag are small molecules that can be administered orally on a once-daily schedule $[2,3]$. All three TPO-RAs are well tolerated with response rate defined as platelet count over 50x109/L during a finite period of time, of $60-90 \%$. Most ITP subjects will maintain a steady platelet count with improvement of bleeding symptoms while they continue TPO-RA therapy. However, when TPO-RAs are stopped, the platelet counts will return to base-line levels. As TPO-Ras treatment is quite expensive, prolonged use will create a significant financial burden on the health care system or patients. Recently, there were increasing reports of treatment-free durable responses after discontinuation of thrombopoietin receptor agonist. Mahevas et al [4] studied 54 adults ITP patients treated with at least one Tpo-RA over a 5-year period in France. TPO-RAs were discontinued in 20 patients who achieved a complete response and eight patients showed a mean treatment free response of 13.5 months (range 5-27 months), giving an overall offtherapy sustained response rate of $14.8 \%$ (8/54). Gonzales et al [5] evaluated treatment free response after discontinuing eltrombopag in 49 out of a cohort of 260 chronic ITP patients. Among these 49 patients, 26 maintained a platelet count $\geq 100 \times 10^{9} / \mathrm{L}$ for at least 6 months after stopping eltrombopag without any additional ITP therapy, while 23 relapsed giving an overall sustained response rate of $10 \%(26 / 260)$. These 26 subjects were all chronic ITP patients who had received an average of 4 (range 2-5) prior therapies. In another study [6], Gonzales et al studied 30 newly diagnosed ITP, 30 persistent
ITP and 160 chronic ITP subjects for treatment free response after discontinuing eltrombopag. Five newly diagnosed (16.6\%), four persistent (13.3\%) and thirty-five chronic (22\%) ITP were able to achieve off therapy response of $\geq 6$ months. In another study of adult ITP treated with romiplostim by Newland et al, 75 subjects with ITP of $\leq 6$ months and who had received romiplostim for $\leq 12$ months have treatment discontinued and treatment-free durable responses (platelet counts $>50 \times 10^{9} / \mathrm{L}$ lasting at least six months) was observed in $23(32 \%)$ subjects [7].

Zhang et al [8] conducted a pilot study to evaluate whether a 12-week course of eltrombopag plus 1-3 courses of pulsed dexamethasone as first line therapy for ITP patients could increase the proportion of patients with platelets $>50 \times 10^{9} / \mathrm{L}$ off therapy at 6 months. Eligible subjects had confirmed ITP and platelet counts $<30 \times 10^{9} / \mathrm{L}$ or platelet counts $<50 \times 10^{9} / \mathrm{L}$ and significant bleeding symptoms (WHO bleeding scale 2 or above). Patients must have no prior ITP treatment. Treatment consisted of eltrombopag 25$75 \mathrm{mg}$ daily according to platelet response for 12 weeks plus pulsed dexamethasone, $40 \mathrm{mg}$ daily for 4 consecutive days every 4 weeks for 1-3 courses. Fifty subjects with primary ITP were successfully enrolled from November 2014 to January 2019. In an intention to treat analysis, $26 / 50$ (52\%) had achieved the primary endpoint of platelet counts > $50 \times 10^{9} / \mathrm{L}$ for more than 6 months after discontinuation of treatment. Three subjects had withdrawn consent before starting treatment, one was withdrawn because of protocol violations and one subject subsequently was diagnosed amegakaryocytic thrombocytopenia. Excluding these 5 subjects, the treatment free response rate would be $57.7 \%$. Among these 26 patients, 17 had maintained platelet count over $100 \times 10^{9} / \mathrm{L}$ for longer than 18 months (mean 41.1, range 19-55 months). Age, sex, clinical features, average daily dose of eltrombopag and number of courses of dexamethasone of these 17 subjects were not significantly different from the rest of the subjects. Among remaining 9 subjects, three had relapsed with satisfactory response to 4 - week course of eltrombopag plus pulsed dexamethasone. The 
remaining six had platelet counts fluctuating between $40-60 \mathrm{x} \times 10^{9} / \mathrm{L}$ without any significant bleeding symptoms or further ITP treatment (manuscript submitted). Even though this was only a single arm study, the treatment free response rate was the highest reported so far. Also, the mean duration of the treatment free response was also among the longest reported. These data suggested that 12 -week of eltrombopag plus dexamethasone as first line treatment may result in prolonged response off therapy in nearly half of ITP subjects.

Treatment free prolonged response can be achieved in $10-50 \%$ of ITP subjects treated with TPORAs. We suggested that ITP subjects who have achieved a steady platelet counts of $>50 \times \mathrm{x} 10^{9} / \mathrm{L}$ with minimal bleeding symptoms after 3 or more months of treatment with a stable dose of TPORAs should have gradually tapering of the TPORAs every 2 weeks the minimum amount required to maintain an adequate platelet count for minimal bleeding symptoms. In $10-30 \%$ of ITP subjects, the TPORAs can be tapered off to achieve treatment free prolonged response. In treatment naïve ITP subjects, eltrombopag plus pulsed dexamethasone is a promising treatment choice for achieving treatment free response.

\section{References}

1. Amgen I Nplate (2017) romiplostim. Thousand Oaks, CA: Amgen.

2. Glaxo-Smith-Kline Promacta (2017) eltrombopag. NC: Research Triangle Park.

3. Pharmaceuticals D Doptelet (2018) avatrombopa. tablets: US prescribing information.

4. Mahevas M, Fain O, Ebbo M, et al. (2014) The temporary use of thrombopoietinreceptor agonists may induce a prolonged remission in adult chronic immune thrombocytopenia. Results of a French observational study. $\mathrm{Br} J$ Haematol.165:865-869.

5. Gonzalez-Lopez TJ, Pascual C, Alvarez-Roman MT, et al. (2015) Successful discontinuation of eltrombopag after complete remission in patients with primary immune thrombocytopenia. Am J Hematol 90: E40-43.

6. Gonzalez-Lopez TJ, Fernandez-Fuertes F, Hernandez-Rivas JA, et al.(2019) Efficacy and safeof eltrombopag in persistent and newly diagnosed ITP in clinical practice. International Journal of Hematology 106: 508-516.

7. Newland A, Godeau B, Priego V, et al. (2016) Remission and platelet responses with romiplostim in primary immune thrombocytopenia: final results from a phase 2 study. Br J Haematol 172:262-273.

8. Zhang L, Zhang M, Du X, Cheng Y, and Cheng G (2020) Safety and efficacy of eltrombopag plus pulsed dexamethasone as first-line therapy for immune thrombocytopenia. British Journal of Hematology. 